\title{
Medium-chain triglycerides/long-chain triglycerides versus long-chain triglycerides in treatment of cancer patients with major body mass loss. Survival in patients with refractory cachexia
}

\author{
Jarosław Szefel ${ }^{1,2}$, Wiesław J. Kruszewski ${ }^{1,2}$, Mariusz Szajewski ${ }^{1,2}$, Maciej Ciesielski ${ }^{1,2}$, Ewa Sobczak ${ }^{3}$, \\ Maksymilian Czerepko², Wiesława Łysiak-Szydłowska ${ }^{4}$ \\ ${ }^{1}$ Department of Propaedeutic Oncology, Faculty of Health Sciences, Medical University of Gdansk, Gdynia, Poland \\ 2Department of Surgical Oncology, Gdynia Oncology Centre, PCK’s Maritime Hospital, Gdynia, Poland \\ ${ }^{3}$ Department of Clinical Nutrition, Medical University of Gdansk, Gdansk, Poland \\ ${ }^{4}$ Powislanski College, Faculty of Health Science, Kwidzyn, Poland
}

Gastroenterology Rev 2016; 11 (3): 181-186

DOI: $10.5114 / p g .2016 .57734$

Key words: medium-chain triglycerides, long-chain triglycerides, refractory cachexia, survival time.

Address for correspondence: Jarosław Szefel MD, PhD, Department of Propaedeutic Oncology, Faculty of Health Sciences,

Medical University of Gdansk, 9b Powstania Styczniowego, 81-519 Gdynia, Poland, phone: +48 509485 357, e-mail: jaszefel@mp.pl

\begin{abstract}
Introduction: Currently there are no established guidelines regarding the use of long-chain triglycerides (LCT) vs. medium-chain triglycerides medium-chain triglycerides (MCT)/long-chain triglycerides (LCT) in total parenteral nutrition (TPN). Severe malnutrition of patients with refractory cachexia (RC) often causes their disqualification from invasive methods of treatment thus decreasing their quality of life and survival time.

Aim: To compare the changes in nutritional state of patients with RC receiving PN with LCT and LCT/MCT lipid emulsions and to assess the influence of enteral nutrition on their survival time.

Material and methods: The study group comprised of 50 patients (23 female, 27 male) with a median age of 66 years. Refractory cachexia was diagnosed in them due to dysphagia secondary to solid tumours causing obstruction of the gastrointestinal tract (GT). All patients were qualified for surgical gastrostomy due to contraindications to percutaneous endoscopic gastrostomy. The patients were randomly assigned into one of two groups and perioperatively received either LCT or LCT/MCT. Blood samples were collected four times and tested for: total protein, albumin, prealbumin, and C-reactive protein concentration. Patients received Home Enteral Nutrition after discharge from hospital.

Results: Changes in nutritional status parameters were similar among patients receiving lipid emulsions LCT vs. MCT/LCT in TPN for 11 days. The mean survival time of all patients operated to gain enteral access to nutrition was $192 \pm 268$ days, and the median survival was 98 days.

Conclusions: Regarding the short-term TPN, the results of the study do not demonstrate any superiority of MCT/LCT lipid emulsions over LCT, or vice versa. The inability to eat significantly accelerates unintended body mass loss among patients with RC. Disqualification from invasive treatment options deprives some patients of the benefits they might have obtained from the surgical access to GT and enteral nutrition.
\end{abstract}

\section{Introduction}

Currently, artificial nutrition of patients with refractory cachexia $(\mathrm{RC})$ is not standard in palliative care [1]. Although nutritional support cannot reverse the body mass loss (BML) in this advanced stage, it can slow the rate of BML and improve the patient's physical abilities and quality of life $[2,3]$. It is accepted that the survival time of palliative care patients is less than 3 months, while for terminal care patients it is less than 1 month. Therefore, the decisions regarding these patients' nutritional and hydration support must be made individually $[4,5]$. Improving the nutritional state is a key element of palliative care, with the aim to alleviate the disease symptoms and increase the survival time of oncological patients $[6,7]$.

The European Society for Clinical Nutrition and Metabolism (ESPEN) guidelines do not recommend nutri- 
tional therapy of cancer patients who have an intact ability to eat [8]. This recommendation is general and does not apply to patients who are unable to eat due to gastrointestinal tract (GIT) obstruction or semi-obstruction. Such patients require procedures that allow enteral nutrition (EN), e.g. stent in the area of the tumour, percutaneous endoscopic gastrostomy (PEG) placement, or percutaneous ileostomy. If such procedures are not technically possible or contraindicated, the patients can be offered a tumour bypass operation, surgical gastrostomy, or feeding jejunostomy below the tumour. Undernutrition, dehydration, and advanced tumour increase the risk of post-operative complications, although this can be reduced by using parenteral nutrition (PN) for 7-10 days pre-operatively [9]. Immunonutrition may boost the reduced immune response of such patients [10]. Unfortunately, the results of clinical trials regarding n-3 polyunsaturated fatty acid (PUFA) supplementation are equivocal [11-13].

\section{Aim}

Our study had two aims: to compare the changes in nutritional state of patients with $R C$ receiving $P N$ with long-chain triglycerides (LCT) and LCT/medium-chain triglycerides (MCT) lipid emulsions and to assess the influence of EN on their survival time.

\section{Material and methods}

This clinical trial included 50 patients (23 female, 27 male), median age $65 \pm 11$ years, with GIT cancer and RC. In terms of BML, body mass index (BMI), age, and sex there were no statistically significant differences between the patients in the LCT vs. MCT/LCT groups (Table I).

Inclusion criteria:

- Cancer diagnosis confirmed by pathological report,

- Disqualified from oncological treatment due to advanced stage of cancer and/or poor overall condition, - Indications for nutritional support defined by Nutritional Risk Score (NRS 2002) $\geq 3$ points,

- Primary or secondary tumour or metastasis causing a GIT obstruction or semi-obstruction that prevents meeting the protein-energy demand via oral route,

Table I. Demographic data of both study arms

\begin{tabular}{lccc} 
Parameter & All patients & MCT/LCT & LCT \\
\cline { 2 - 4 } & & $\boldsymbol{x} \pm$ SD \\
\hline Age [years] & $66 \pm 11$ & $65 \pm 9$ & $66 \pm 12$ \\
\hline $\begin{array}{l}\text { Gender (\% female } \\
\text { to \% male) }\end{array}$ & $46 / 54$ & $50 / 50$ & $43 / 57$ \\
\hline BMI [kg/m²] & $21 \pm 5$ & $22 \pm 5$ & $20 \pm 4$ \\
\hline $\begin{array}{l}\text { BML (\% in the } \\
6 \text { months pre-op) }\end{array}$ & $21 \pm 9$ & $22 \pm 9$ & $21 \pm 9$ \\
& & &
\end{tabular}

- Contraindications to or technical problems with PEG tube placement or other minimally-invasive methods of accessing the GIT,

- Open surgery as the only means of restoring enteral feeding (via gastrostomy or feeding jejunostomy, exploratory laparotomy, colostomy, or anastomosis bypassing the obstruction).

Exclusion criteria:

- Contraindications to general anaesthesia,

- Active inflammatory process (e.g. pneumonia, urinary tract infection),

- Comorbidities causing significant metabolic disturbances (e.g. diabetes, liver cirrhosis).

On the day of admission, each patient had a central venous access placed while in the operating room. Next, the patients were assigned into one of two groups and perioperatively received either LCT emulsion $(n=30)$ or LCT/MCT (50: 50) $(n=20)$. Patients who were included in this study were not informed which lipid emulsion they will receive. The emulsions were assigned to the patients using an online random number generator (http://www.randomizer.org/). The researchers knew, but had no influence on the type of administered substance. Based on the randomly generated list, patients received the mixture based on " 0 " corresponding to the LCT mixture and " 1 " to MCT/LCT.

TPN was administered for 5 days pre-op, withdrawn on the day of operation, and then re-administered postop (modified according to the increase of EN and decrease of PN, with constant total amount of kcal per day).

Blood samples ( $2 \mathrm{ml}$ each) were collected from the patients' ulnar veins in this sequence:

$-1^{\text {st }}$ sample - during the $1^{\text {st }}$ day of hospitalisation,

$-2^{\text {nd }}$ sample $-6^{\text {th }}$ day of hospitalisation ( $5^{\text {th }}$ day of TPN), $-3^{\text {rd }}$ sample $-7^{\text {th }}$ day of hospitalisation ( $1^{\text {st }}$ day post-op, $6^{\text {th }}$ day of TPN),

$-4^{\text {th }}$ sample $-11^{\text {th }}$ day of hospitalisation.

The following blood parameters were measured: albumin, prealbumin, lymphocytes, total protein, and C-reactive protein (CRP).

\section{Statistical analysis}

Patient mortality was determined by accessing the official governmental death registry (44 of 50 patients). Statistical analysis was performed using Statistica software (version 10, StatSoft; Tulsa, OK). The influence of particular lipid emulsions on the examined clinical markers (blood samples 1-4) was analysed using the sign test for paired data. The correlations between the markers in both patient groups were measured using the Mann-Whitney $U$ test. Survival was analysed with Kaplan Meier and log rank tests. Value of $p<0.05$ was set as statistically significant. 


\section{Ethics}

Permission of the Independent Bioethics Committee for Scientific Research at the Medical University of Gdansk was obtained prior to the start of this study. All patients were notified about the aims of this study and gave their informed consent to participate. Due to ethical concerns, this study did not include a control group in nobody underwent surgical intervention, despite having possible benefit from surgical access to the GIT.

This study began in 2008. Initially it was designed to include 100 patients. In 2009 ESPEN published recommendations stating that undernourished patients should receive nutritional support for at least 10 days prior to surgery. As a result, starting in 2010, patients received nutrition support for at least 10 days and the study was concluded.

\section{Results}

In $78 \%$ of the patients the passage of food was obstructed by a primary GIT tumour, whereas in the remaining $22 \%$ by infiltration or metastasis from other organs (Table II).

In all patients the diagnosis of cancer was made based on histopathological testing. In $8 \%$ of the patients it was not possible to locate the primary focus of cancer and the diagnosis was made based on samples of metastatic tissue. In $67 \%$ of the patients the ECOG/WHO/Zubrod score was assessed at 4 points and in 33\% it was 3 points [14]. The following surgical procedures were performed: gastrostomy or feeding jejunostomy (58\%), anastomosis bypassing the obstruction (20\%), colostomy (12\%), palliative
Table II. Location of cancer

\begin{tabular}{lcc} 
Location of cancer & & $\boldsymbol{N}$ \\
\hline GIT & Stomach & 21 \\
\cline { 2 - 3 } & Oesophagus & 7 \\
\cline { 2 - 3 } & Colon & 6 \\
\cline { 2 - 3 } & Rectum & 3 \\
\hline Non-GIT & Pancreas & 1 \\
\hline Tongue & 1 \\
\cline { 2 - 3 } & Lung & 3 \\
\cline { 2 - 3 } & Ovary & 1 \\
\cline { 2 - 3 } & Testicle & 1 \\
\hline Larynx & 1 \\
\cline { 2 - 3 } & Breast & 1 \\
\cline { 2 - 2 } & Unknown & 4 \\
\cline { 2 - 2 } & Total & 50
\end{tabular}

gastrectomy (1\%), and exploratory laparotomy in the remaining.

\section{The relationship between the studied parameters and survival of patients}

The mean post-op survival time of all patients was $192 \pm 268$ days and the median was 98 days. Fifty-seven percent of studied patients survived $>90$ days. The analysis showed that there is a statistical relationship only between CRP $(p<0.007)$ and the probability of survival over 90 days, but there is no relationship between the parameters of nutritional status and the probability of survival over 90 days (Figures 1 and 2).

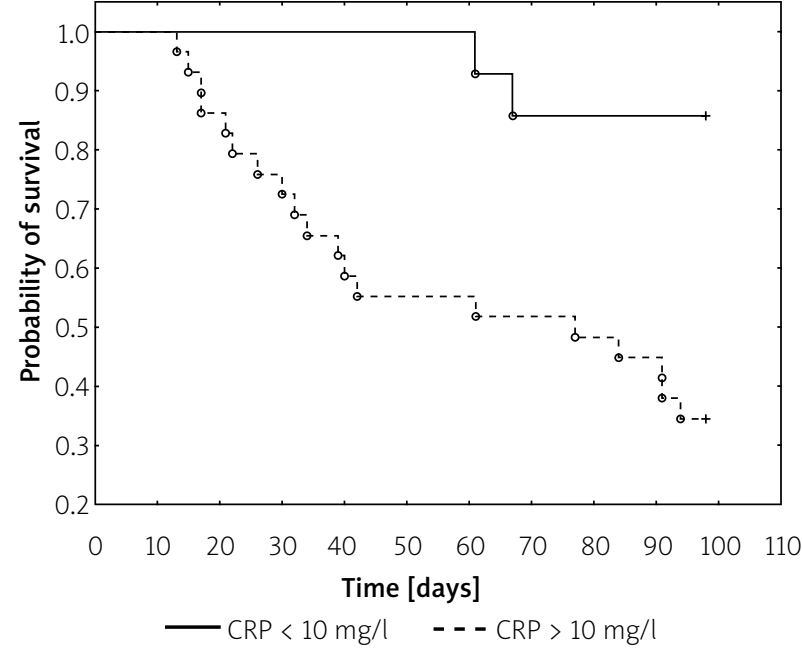

Figure 1. The Kaplan-Meier survival estimates for the patients with CRP $<10 \mathrm{mg} / \mathrm{l}$ and CRP > $10 \mathrm{mg} / \mathrm{l}$ on the day of admission

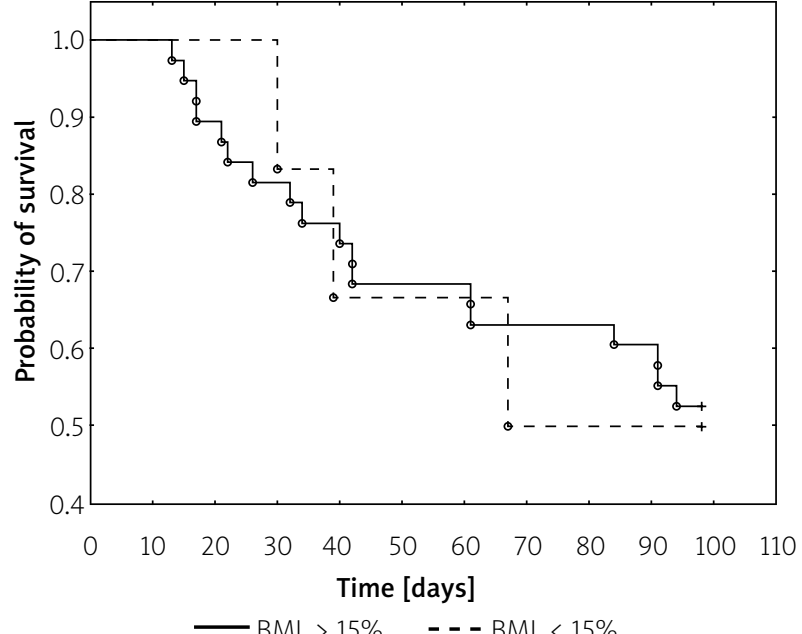

Figure 2. The Kaplan-Meier survival estimates for the patients with $\mathrm{BML}<10 \%$ and $\mathrm{BML}>10 \%$ in the 6 months prior to hospitalisation 
Table III. Changes in the nutrition state markers of patients receiving lipid emulsions MCT/LCT or LCT between the $1^{\text {st }}$ and $4^{\text {th }}$ sample

\begin{tabular}{|c|c|c|c|c|}
\hline \multirow[t]{4}{*}{ Marker } & \multicolumn{4}{|c|}{ Difference between the $1^{\text {st }}$ and $4^{\text {th }}$ sample } \\
\hline & \multicolumn{2}{|c|}{ MCT/LCT } & \multicolumn{2}{|c|}{ LCT } \\
\hline & $1^{\text {st }}$ & $4^{\text {th }}$ & $1^{\text {st }}$ & $4^{\text {th }}$ \\
\hline & $x \pm \mathrm{SD}$ & $x \pm \mathrm{SD}$ & $x \pm \mathrm{SD}$ & $x \pm S D$ \\
\hline Total protein [g/l] & $61 \pm 12$ & $55 \pm 7^{\star}$ & $64 \pm 9$ & $56 \pm 8^{\#}$ \\
\hline Albumin $[\mathrm{g} / \mathrm{l}]$ & $32 \pm 6$ & $27 \pm 7^{*}$ & $33 \pm 5$ & $27 \pm 5^{\#}$ \\
\hline Prealbumin $[\mathrm{mg} / \mathrm{l}]$ & $224 \pm 167$ & $203 \pm 108$ & $186 \pm 122$ & $148 \pm 135$ \\
\hline Lymphocytes $\left[\times 10^{9} / 1\right]$ & $1.1 \pm 0.6$ & $1.1 \pm 0.5$ & $1.3 \pm 0.7$ & $1.3 \pm 0.8$ \\
\hline $\mathrm{CRP}[\mathrm{mg} / \mathrm{l}]$ & $33 \pm 41.3$ & $89 \pm 50^{\#}$ & $60 \pm 72$ & $90 \pm 77^{*}$ \\
\hline
\end{tabular}

${ }^{*} p<0.05,{ }^{*} p<0.005$

The influence of MCT/LCT emulsions vs. LCT on changes in the concentrations of markers of nutritional status

In both patient groups the levels of nutrition status markers significantly decreased between the time of $1^{\text {st }}$ and $4^{\text {th }}$ blood sample collections (Table III). The extent of this decrease was similar in both groups. The CRP levels increased significantly but more so in the MCT/ LCT group $(p<0.042)$.

\section{Discussion}

The EPCRC (European Palliative Care Research Collaborative) emphasises that the reversal of BML in patients in the RC stage is not possible, regardless of the type and contents of nutritional mixture administered [15]. Blum et al. suggested specific criteria to distinguish particular stages of cancer cachexia and defined RC as $\mathrm{BWL}>15 \%$ in the last 6 months and $\mathrm{BMI}<23 \mathrm{~kg} / \mathrm{m}^{2}$ or BWL $>20 \%$ in the last 6 months and BMI $<27 \mathrm{~kg} / \mathrm{m}^{2}$ [16]. However, our results do not support their concepts.

The BML of patients with solid tumour of the GIT causing obstruction/semi-obstruction is a result not of cancer cachexia alone, but of both cancer cachexia and simple undernutrition [17]. Due to heavy reliance on BML, these patients may be too hastily diagnosed as being in the RC stage. That in turn may lead to depriving these patients of potentially beneficial surgical restoration of EN. A commonly accepted mean survival time of palliative care patients is 90 days. Whereas the mean survival time of the patients in our study was 192 days thanks to the surgical GIT access allowing EN. The lack of correlation between BML and expected survival time $>90$ days suggests individual consideration of these patients [18, 19].

Selection of the optimal contents of a nutritional mixture and artificial diet for cancer cachexia patients is still an unsolved problem. It is recommended that the lipids/glucose ratio in the nutrition of oncological patients be increased; however, these recommendations do not specify which fatty acids [20]. Long-chain fatty acids (LCFA) require L-carnitine as a transporter into the mitochondrial matrix, while the MCT do not. The L-carnitine concentration in the skeletal muscles and serum is reduced below reference values in CC patients due to the rate of skeletal muscle degradation (secondary to reduced dietary intake of L-carnitine) and loss in urine [21]. The serum L-carnitine deficiency is replenished from muscle storage after some delay [22]. In theory, mixing LCT and MCT lipid mixtures should improve the energy efficiency of fatty acid metabolism. However, our results and previous literature do not support this hypothesis [23-25].

The heart and skeletal muscles contain $98 \%$ of the body's L-carnitine, which in a 70-kg male amounts to about $100 \mathrm{mmol}$. Taking into consideration the lack of dietary intake and 24-hour loss in urine, that amount of L-carnitine should suffice for about 660 days [26, 27]. It is possible that the L-carnitine pool ensures that the $\beta$-oxidation of LCFA takes place in muscles for a longer time than the expected survival time of RC patients. This may be the reason why there is no difference between the influence of MCT/LCT and LCT-only mixtures on the nutritional markers. The immune system cells do not store L-carnitine but gather it from the serum. That is why in state of reduced serum L-carnitine, the MCT/LCT emulsions may provide the immune cells with more energy than LCT-only and improve their response to inflammation [28].

\section{Conclusions}

Our results do not indicate the superiority of use of MCT/LCT or LCT lipid emulsions in PN for patients 
with cancer cachexia. Simple undernutrition plays an important role in premature RC recognition in patients with a tumour causing gastrointestinal obstruction or semi-obstruction. The BML lacks prognostic value regarding the survival time in this patient group. Such patients require an individual treatment approach because some of them might benefit from invasive methods of restoring feeding. The lack of verified markers makes it difficult to distinguish the various stages of cachexia in clinical practice.

\section{Acknowledgments}

Financial support for this project was provided by Fundacja na Rzecz Rozwoju Chirurgii “Viribus Unitis” im. Oskara Jankaua.

\section{Conflict of interest}

The authors report no conflicts of interest.

\section{References}

1. WHO: WHO Definition of Palliative Care. [(accessed on 21 December 2012)]. Available online: http://www.who.int/cancer/ palliative/definition/en/.

2. Fearon K, Strasser F, Anker SD, et al. Definition and classification of cancer cachexia: an international consensus. Lancet Oncol 2011; 12: 489-95.

3. Marin Caro MM, Laviano A, Pichard C. Nutritional intervention and quality of life in adult oncology patients. Clin Nutr 2007; 26: 289-301.

4. Bachmann P, Marti-Massoud C, Blanc-Vincent MP, et al. Summary version of the Standards, Options and Recommendations for palliative or terminal nutrition in adults with progressive cancer (2001). Br J Cancer 2003; 89 Suppl. 1: S107-10.

5. Oh do Y, Kim JH, Lee SH, et al. Artificial nutrition and hydration in terminal cancer patients: the real and the ideal. Support Care Cancer 2007; 15: 631-6.

6. Bozzetti F. Nutritional supplementation in advanced cancer patients: Re: "Influence of a nutritional intervention on dietary intake and quality of life in cancer patients". Nutrition 2014; 30: $957-8$.

7. Uster A, Ruefenacht U, Ruehlin M, et al. Influence of a nutritional intervention on dietary intake and quality of life in cancer patients: a randomized controlled trial. Nutrition 2013; 29: 1342-9.

8. Bozzetti F, Arends J, Lundholm K, et al. ESPEN Guidelines on parenteral nutrition: non-surgical oncology. Clin Nutr 2009; 28: 445-54.

9. Braga M, Ljungqvist O, Soeters P, et al. ESPEN Guidelines on parenteral nutrition: surgery. Clin Nutr 2009; 28: 378-86.

10. Klek S, Szybinski P, Szczepanek K. Perioperative immunonutrition in surgical cancer patients: a summary of a decade of research. World J Surg 2014; 38: 803-12.

11. Sorensen LS, Thorlacius-Ussing O, Rasmussen HH, et al. Effects of perioperative supplementation with omega- 3 fatty acids on leukotriene $\mathrm{B}(4)$ and leukotriene $\mathrm{B}(5)$ production by stimulated neutrophils in patients with colorectal cancer: a randomized, placebo-controlled intervention trial. Nutrients 2014; 6: 4043-57.

12. Pradelli L, Mayer K, Muscaritoli M, Heller AR. n-3 fatty acid-enriched parenteral nutrition regimens in elective surgical and ICU patients: a meta-analysis. Crit Care 2012; 16: R184.

13. Umpierrez GE, Spiegelman R, Zhao V, et al. A double-blind, randomized clinical trial comparing soybean oil-based versus olive oil-based lipid emulsions in adult medical-surgical intensive care unit patients requiring parenteral nutrition. Crit Care Med 2012; 40: 1792-8.

14. Oken MM, Creech RH, Tormey DC, et al. Toxicity and response criteria of the Eastern Cooperative Oncology Group. Am J Clin Oncol 1982; 5: 649-55.

15. Radbruch L, Elsner F, Trottenberg P, et al. Clinical practice guidelines on cancer cachexia in advanced cancer patients. Department of Palliative Medicinen/European Palliative Care Research Collaborative; Aachen 2010.

16. Blum D, Stene GB, Solheim TS, et al. Validation of the Consensus - Definition for cancer cachexia and evaluation of a classification model: a study based on data from an international multicentre project (EPCRC-CSA). Ann Oncol 2014; 25: $1635-42$.

17. Kubrak $\mathrm{C}$, Olson $\mathrm{K}$, Jha $\mathrm{N}$, et al. Nutrition impact symptoms: key determinants of reduced dietary intake, weight loss, and reduced functional capacity of patients with head and neck cancer before treatment. Head Neck 2010; 32: 290-300.

18. Martin L, Senesse P, Gioulbasanis I, et al. Diagnostic criteria for the classification of cancer-associated weight loss. J Clin Oncol 2015; 33: 90-9.

19. Brookman-May S, Kendel F, Hoschke B, et al. Impact of body mass index and weight loss on cancer-specific and overall survival in patients with surgically resected renal cell carcinoma. Scand J Urol Nephrol 2011; 45: 5-14.

20. Korber J, Pricelius S, Heidrich M, Muller MJ. Increased lipid utilization in weight losing and weight stable cancer patients with normal body weight. Eur J Clin Nutr 1999; 53: 740-5.

21. Szefel J, Kruszewski WJ, Ciesielski M, et al. L-carnitine and cancer cachexia. I. L-carnitine distribution and metabolic disorders in cancer cachexia. Oncol Rep 2012; 28: 319-23.

22. Sachan DS, Dodson WL. The serum carnitine status of cancer patients. J Am Coll Nutr 1987; 6: 145-50.

23. Ulrich H, Pastores SM, Katz DP, Kvetan V. Parenteral use of medium-chain triglycerides: a reappraisal. Nutrition 1996; 12 : 231-8.

24. Chambrier C, Bannier E, Lauverjat M, et al. Replacement of long-chain triglyceride with medium-chain triglyceride/longchain triglyceride lipid emulsion in patients receiving longterm parenteral nutrition: effects on essential fatty acid status and plasma vitamin K1 levels. JPEN J Parenter Enteral Nutr 2004; 28: 7-12.

25. Martin-Pena G, Culebras JM, De I P, et al. Effects of 2 lipid emulsions (LCT versus MCT/LCT) on the fatty acid composition of plasma phospholipid: a double-blind randomized trial. JPEN J Parenter Enteral Nutr 2002; 26: 30-41.

26. Sayed-Ahmed MM. Role of carnitine in cancer chemotherapy-induced multiple organ toxicity. Saudi Pharm J 2010; 18: 195-206. 
27. Fornasini G, Upton RN, Evans AM. A pharmacokinetic model for L-carnitine in patients receiving haemodialysis. $\mathrm{Br} J \mathrm{Clin}$ Pharmacol 2007; 64: 335-45.

28. Szefel J, Kruszewski WJ, Ciesielski M, et al. L-carnitine and cancer cachexia. II. Effects of lipid emulsion used in total parenteral nutrition on parameters of hemostasis and inflammatory state in L-carnitine deficiency in myocytes. Oncol Rep 2012; 28: 324-9.

Received: 13.04.2015

Accepted: 11.05 .2015 